\title{
Análise da contribuição de mapeamento tecnológico (Roadmapping) para a gestão estratégica da inovação: estudo de caso de uma multinacional do setor automotivo
}

\author{
Analysis of the contribution of technological mapping \\ (Roadmapping) for the strategic innovation \\ management: case study of a multinational \\ automotive industry
}

\author{
Débora Guimarães Sousa ${ }^{1}$ \\ Rodrigo Baroni Carvalho \\ José Márcio Castro ${ }^{3}$
}

\begin{abstract}
Resumo
O objetivo do artigo é analisar a contribuição da metodologia de mapas tecnológicos (Roadmapping) para o processo de inovação tecnológica em uma organização multinacional do setor automotivo. Foi utilizado um estudo de caso único com observação participante, análise documental e entrevistas semiestruturadas com treze profissionais de diversas áreas que participaram do processo de construção do roadmap. Os resultados apontaram que a ferramenta auxiliou o planejamento estratégico e que as contribuições para a inovação tendem a ser evidenciadas à medida que as ações que surgiram do
\end{abstract}

Mestre em Administração. Pontifícia Universidade Católica de Minas Gerais (PUCMinas). Belo Horizonte, MG, Brasil. ORCID: deboraguimaraesbh@gmail.com

Doutor em Ciência da Informação. Pontifícia Universidade Católica de Minas Gerais (PUCMinas). Belo Horizonte, MG, Brasil. baroni@pucminas.br

Doutor em Administração pela Faculdade de Economia e Administração da Universidade de São Paulo/FEA/USP. Pontifícia Universidade Católica de Minas Gerais (PUCMinas). Belo Horizonte, MG, Brasil. josemarcio@pucminas.br

Como citar:

Sousa, D. G., Carvalho, R. B., \& Castro, J. M. (2020). Análise da contribuição de mapeamento tecnológico (Roadmapping) para a gestão estratégica da inovação: estudo de caso de uma multinacional do setor automotivo. Revista Inovação, Projetos e Tecnologias, 8(1), 13-24.

https://doi.org/10.5585/iptec.v8i1.17400 
roadmapping sejam concluídas. O caso estudado evidenciou o potencial do roadmapping em alinhar as perspectivas da estratégia e inovação em um setor caracterizado por intensa evolução tecnológica.

Palavras-chave: Roadmapping; Setor automotivo; Gestão da Inovação Tecnológica; Planejamento Estratégico.

\begin{abstract}
The objective of the paper is to analyze the contribution of the technology roadmapping methodology (TRM) to the process of technological innovation in a multinational automobile industry. A single case study approach was used with participant observation, document analysis and semi-structured interviews with thirteen professionals from different areas who participated in the roadmap process. The results showed that TRM has support the strategic planning and that the innovation contributions tend to emerge along with the deployment of TRM-inspired initiatives. The case has given evidences of the TRM potential to strategy and innovation alignment with an industry characterized by intense technological evolution.
\end{abstract}

Keywords: Roadmapping; Automobile industry; Technological Innovation Management; Strategic Planning.

\title{
1 Introdução
}

Rápidas mudanças tecnológicas e ciclos de produtos mais curtos têm aumentado a dificuldade de se gerenciar processos de inovação. O equilíbrio entre empurrar a tecnologia (technology push) e buscar as demandas do mercado (marketing pull) é complexo e pode variar consideravelmente ao longo do ciclo da indústria. Em muitos casos, é somente quando uma tecnologia de base se desenvolve é que as suas possibilidades de aplicação emergem, possibilitando novas utilizações e despertando a atenção de clientes. Neste estágio, o mercado desempenha um papel importante de dirigir o ritmo e a direção da mudança tecnológica. No entanto, o processo de combinar a capacidade tecnológica com as necessidades do mercado continua a ser fundamental para o sucesso (Rothwell, 1994) e, nesses termos, constata-se que a tecnologia tem desempenhado cada vez mais um papel fundamental no processo de inovação.

No contexto da gestão da inovação, as organizações têm buscado utilizar ferramentas e metodologias que auxiliem na análise de cenários, na inteligência competitiva e na prospecção de tecnologias, sendo que o roadmapping tem apresentado um uso crescente. Muitos tipos e métodos de previsão tecnológica foram desenvolvidos nas últimas três décadas, mas dentre todos, o roadmap de tecnologia se destaca como o mais popular, sendo amplamente utilizado para apoiar o desenvolvimento de tecnologias futuras (Hussain et al., 2017). Segundo Phaal, Farrukh e Probert (2010), o roadmapping é uma ferramenta de estrutura de negócio que permite visualizar a evolução de um determinado assunto a ser explorado, dando apoio à inovação de caráter estratégico. Um dos diferenciais da ferramenta é sua capacidade de permitir uma visualização integrada do mercado, dos produtos e da tecnologia de um determinado assunto. O roadmapping foi disseminado por Willyard e McClees (1987) na Motorola, sendo que os autores mais referenciados no tema são Phaal, Farruck e Probert, a partir da década de 2000. Em uma revisão sistemática recente da literatura sobre TRM (Technology Roadmapping), Alcântara e Martens (2019) reforçam a constatação de que o professor Robert Phall da Universidade de Cambridge é o pesquisador mais ativo na temática, sendo seus trabalhos adotados como referência central desta pesquisa.

Em contextos em que a centralidade da tecnologia é premente, faz-se necessário ter ferramentas que auxiliem o processo de desdobramento da estratégia em tendências e vetores tecnológicos que norteiem a gestão da inovação. Nas grandes organizações, pode haver tantas tecnologias usadas em diferentes áreas de negócios que até os engenheiros podem não estar cientes de todas elas (Alcântara e Martens, 2019). O roadmapping pode ser usado em diversos 
tipos de indústria, e neste artigo sua aplicação foi analisada em uma indústria automobilística multinacional, atendendo a sugestão de novos estudos proposta por Hussain et al.(2017), para quem a aplicação do roadmap precisa ser investigada em outros locais em que os participantes representam vários grupos constituintes, organizações, setores e disciplinas, mas compartilham um interesse comum na adoção de uma tecnologia inovadora.

No setor automotivo, a acumulação tecnológica é usualmente conseguida por meio de design, construção e operação de sistemas complexos. Tal contexto demanda que as indústrias do setor automobilístico utilizem ferramentas gerenciais, tais como o roadmapping, para aprimorar os processo de inovação tecnológica e aplicar a tecnologia com base em seu know-how.

Segundo o anuário mais recente da Associação Nacional dos Fabricantes de Veículos Automotores (ANFAVEA, 20020), considerando os produtos automóveis, comerciais leves, caminhões, ônibus, tratores e outros, a indústria automotiva brasileira emprega direta e indiretamente 1,3 milhão de pessoas. A indústria obteve um faturamento de US\$ 61,9 bilhões em 2018, sendo que sua participação no PIB (Produto Interno Bruto) foi de 3\% no global e 18\% da indústria de transformação, refletindo a importância do setor para a economia nacional. A geração agregada de tributos em 2018 foi de $\mathrm{R} \$ 65,6$ bilhões, O Brasil é o $6^{\circ}$ mercado interno e o $8^{\circ}$ produtor mundial de veículos (ANFAVEA, 2020).

Considerando o papel estratégico do setor automobilístico para a economia brasileira, é relevante analisar a aplicação de metodologias que unifiquem as análises de mercado, de produto e de tecnologia, caracterizando assim um cenário típico para o emprego roadmapping. Portanto, o objetivo do artigo é analisar a contribuição da metodologia de mapeamento tecnológico (roadmapping) para a gestão da inovação, a partir da percepção do uso dos seus praticantes em uma organização multinacional do setor automotivo. Para além desta introdução, o artigo está estruturado do seguinte modo: a seção 2 compreende o referencial teórico com a temática da gestão da inovação e a apresentação da ferramenta de roadmapping; a seção 3 descreve os procedimentos metodológicos e a unidade empírica de análise; a seção 4 detalha o passo-a-passo na aplicação prática do roadmapping; a seção 5 analisa os resultados da pesquisa e sintetiza os depoimentos dos entrevistados por meio de categorias graficamente representadas; a seção 6 sumariza as principais contribuições do trabalho, evidenciando suas limitações e propondo trabalhos futuros.

\section{Fundamentação teórica}

Tidd, Bessant e Pavitt (2008) destacam que é um desafio ter a definição estratégica para desenvolver a trajetória tecnológica da empresa que consiga abordar a diversidade empresarial e setorial. A ferramenta de roadmapping se propõe justamente a preencher esse espaço de construção de uma estratégia tecnológica que oriente a inovação.

Kostoff e Schaller (2001) descrevem o processo de roadmapping como a prática de elaboração de roadmap, que é um mapa com olhar alargado no futuro de um tema definido, sendo realizado com a ajuda das pessoas que conhecem esse tema. Além disso, o roadmap comunica as visões e os recursos que serão necessários para os negócios. De acordo com Phaal et al. (2010), o roadmapping é uma ferramenta de estrutura de negócio que permite visualizar a evolução de um determinado assunto a ser explorado e mapeado, sendo uma ferramenta poderosa e flexível que apoia a inovação e a estratégia. Echols e Petrick (2004) definem o roadmapping como o processo que representa a visão estratégica e tecnológica, tendo sido a primeira ferramenta gerencial que unifica as visões de produto, tecnologia e estratégia. 
Bray e Garcia (1997) destacam que o roadmapping se torna eficaz dentro de uma organização quando é dependente de visão e compromisso dos gestores e funcionários, sendo um processo iterativo e exploratório. Ter um grupo de especialistas discutindo sobre o futuro nas camadas do mapa é importante para a construção do roadmap. A ferramenta permite a convergência de visões de diversas áreas que, por vezes, pouco dialogam como marketing e engenharia.

O roadmap pode ser representado de diversas formas, normalmente inclui representação gráfica e uma linha de tempo estruturada, conforme Figura 1 (Phaal, 2004), que mostra como, ao longo do tempo, podem ser estruturadas as camadas do roadmap (mercado, produto e tecnologia). No entanto, ao construir o roadmap, cada empresa pode fazer suas adaptações contextuas necessárias para melhor utilização da ferramenta.

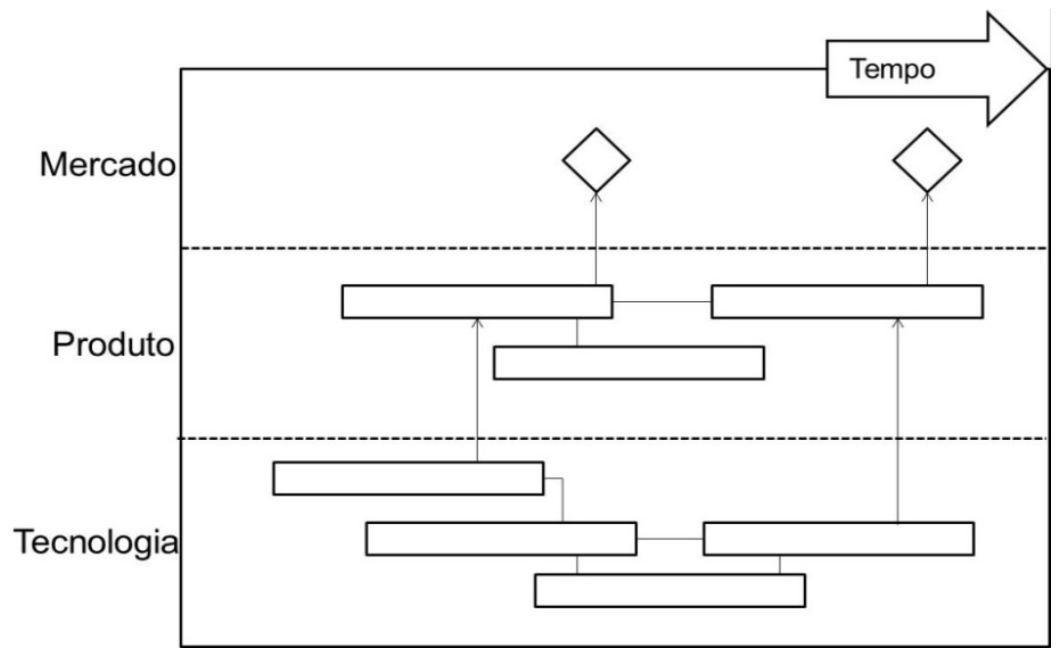

Figura 1: Esquema do Technology Roadmap

Adaptado de Phaal (2004).

Para um bom desenvolvimento do processo de roadmapping é necessário ter desejo de desenvolver processos eficazes, ter as pessoas certas envolvidas e o comprometimento da alta direção. Os fatores que dificultam o roadmapping incluem a sobrecarga de iniciativas, distração de tarefas de curto prazo, falta de dados necessários e o conhecimento não estar disponível (Phaal, Farrukh, \& Probert, 2010).

O roadmapping pode ter uma orientação mais estratégica ou mais tecnológica, sendo esta última o enfoque do presente trabalho. De acordo com Phaal et al. (2007), o strategic roadmapping (SRM) foi projetado para suportar a avaliação estratégica, inovação, oportunidades de negócios e também para alavancar benefícios organizacionais, como: melhoria das decisões de comunicação, construção das equipes de trabalho, desenvolvimento da rede, apoio ao desenvolvimento de processo de negócios. Por sua vez, o processo de technology roadmapping (TRM) envolve identificar, selecionar e desenvolver alternativas tecnológicas que atendam aos produtos, envolvendo também uma equipe de especialistas para organizar e apresentar esse planejamento de forma adequada. Echols e Petrick (2004) também concordam que o technology roadmap liga diretamente a tecnologia às necessidades do negócio, reduzindo a incerteza nos calendários de lançamentos e no planejamento de produtos.

Segundo Phaal et al. (2010), o primeiro passo do roadmapping, é a fase de planejamento quando se deve definir o escopo do projeto, objetivos, recursos, os participantes, treinamento, data e local para os workshops. Após a fase de planejamento, o processo de construção do roadmap 
tecnológico, deve ser dividido em quatro workshops. O primeiro workshop objetiva a construção da camada de marketing, o segundo a camada de produto, o terceiro a de tecnologia e o último visa à integração dessas três camadas (Phaal et al., 2010).

\section{Metodologia de pesquisa}

No âmbito deste trabalho, foi utilizada a abordagem de natureza qualitativa com o método de estudo de caso (Eisenhardt, 1989) para avaliar o uso de roadmapping. A investigação buscou avaliar as conexões do roadmapping com a estratégia e com a inovação tecnológica bem como verificar a sua aplicação na indústria automotiva. A escolha por este procedimento metodológico se baseou também na análise de artigos correlatos sobre roadmapping tais como Abe et al. (2008), Daim e Oliver (2008), Phaal et al. (2001), nos quais foi constatada a utilização intensiva da abordagem de estudos de caso de natureza exploratória.

Do universo de cinquenta participantes das diversas fases do projeto piloto de roadmapping na empresa, foram entrevistadas doze pessoas bem como uma analista de negócios que também utiliza a metodologia roadmapping em outro projeto na empresa. As entrevistas foram realizadas após a finalização dos workshops de construção de mercado, produto, tecnologia e integração. A escolha da amostra de profissionais para a entrevista levou em consideração a participação nos workshops realizados. Foi utilizado como ponto de corte para seleção dos respondentes aqueles que tiveram mais de $30 \%$ de participação dos seis workshops realizados e do treinamento, resultando na predominância de profissionais das áreas de Produto e de Engenharia.

O caso selecionado na indústria automobilística foi a planta industrial situada em Minas Gerais de um grupo automotivo multinacional que adota o roadmapping desde 2012. A planta industrial escolhida é a maior do Brasil e conta com 11 mil funcionários, sendo que cerca de $60 \%$ estão na linha de produção (ANFAVEA, 2020). A escolha do caso se deu por critérios de acessibilidade, da maturidade do uso da ferramenta pela empresa bem como da escassez de estudos sobre roadmapping no setor automotivo.

Eisenhardt (1989) afirma que o estudo de caso tipicamente combina métodos de análise de dados como arquivos, entrevistas, questionários e observação. Nesse trabalho, a coleta de dados foi feita por meio de análise documental, entrevistas semiestruturadas e observação participante do processo de roadmapping do tema Segurança Veicular, uma vez que um dos pesquisadores foi responsável à época pelo projeto na organização estudada. No caso estudado, para melhor triangulação dos dados, adotou-se a observação participante, pois esta se refere a um contexto onde pesquisador e o objeto de pesquisa são sujeitos que interagem e a coleta de dados acontece no próprio ambiente de pesquisa, havendo a possibilidade de se consolidar a realidade, coordenando os fatos e retirando os conceitos que dão sentido a pesquisa (Serva \& Júnior, 1995).

Foram entrevistados gerentes das áreas de Estratégia e Inovação e de Experimentação, supervisor da área de Segurança Veicular, analistas de Segurança Veicular, de Engenharia, de Materiais, de Produto e mais alguns profissionais de áreas correlatas. As entrevistas foram presenciais com roteiro semiestruturado. Foram analisados os seguintes tipos de documentos: roadmaps de organizações especializadas em segurança veicular, cronogramas, relatórios executivos, roadmaps das camadas de mercado, produto e tecnologias bem como relatórios técnicos e apresentações das três camadas. Os dados foram registrados por meio de memorandos e diagramas, ajudando a organizar as categorias de análise por codificação seletiva (Strauss \& Corbin, 2008). 


\section{Aplicação prática da metodologia Roadmapping}

O projeto de roadmapping na empresa em estudo foi liderado pela Gerência de Inovação da empresa com apoio de uma consultoria nacional especializada. A metodologia de roadmapping foi empregada em um projeto piloto, tendo sido escolhido o tema de segurança veicular devido ao interesse da área e também ao fato do assunto ser abordado no programa Inovar Auto, que foi um programa do Governo Federal de incentivo à inovação tecnológica e adensamento da cadeia produtiva de veículos automotores no período de 2013 a 2017. Desde então, a segurança veicular tem sido um dos direcionadores de inovação do setor automotivo.

Tal como recomendado na literatura, o projeto piloto de roadmapping para segurança veicular foi realizado em três grandes fases principais: planejamento, execução e finalização. $\mathrm{Na}$ fase de planejamento, foram feitas a definição do tema e escopo, entrevistas preliminares e treinamento. $\mathrm{Na}$ fase de execução, foram realizados cinco workshops para construção das camadas de mercado, produto e tecnologia. Na fase de finalização, foi feito um workshop para integração das camadas, definição dos projetos a serem executados e o report final.

A fase de planejamento verificou qual seriam as áreas que fariam parte do projeto e qual seria o patrocinador interno do projeto. Também foram realizadas entrevistas com quatro profissionais que são referência interna em segurança veicular para verificar prováveis recortes no contexto e definição do escopo. As entrevistas foram feitas para se ter uma visão rica do tema a fim de alimentar todo o processo, identificar o papel estratégico do assunto na empresa, os principais aspectos envolvidos e a visão de passado-presente-futuro. Todas as entrevistas foram gravadas e compiladas e o resultado apresentado aos envolvidos e utilizados em um treinamento. O treinamento de dois dias foi ministrado pela consultoria responsável pela ferramenta roadmapping para todas as pessoas que seriam envolvidas no decorrer do projeto, visando à imersão nos assuntos segurança veicular e roadmapping. Participaram do treinamento vinte e seis pessoas das seguintes áreas da empresa: engenharia, comercial, exportação, produto e motores e transmissão.

$\mathrm{Na}$ fase de mercado, foi construída a visão de mercado no assunto ao longo do tempo, sendo que em cada subcamada foram registrados os marcos que definem o passado, atividades que já aconteceram no passado e que continuam a interferir no futuro, denominado de passado-presente e futuro do assunto. O entendimento do mercado foi subdividido em dimensões representantes dos requisitos dos clientes, evolução da segurança veicular, posicionamento da empresa e evolução da legislação.

Na fase de produto, analogamente à fase anterior, foram registrados os marcos que definem o produto ao longo do passado, passado-presente e futuro. Esta camada de produto foi subdivida nas partes do veículo como carroceria, chassi, bancos e cinto e também uma específica de produto, para verificar a evolução dos modelos e especificações.

Na fase de tecnologia, foi construída a visão da evolução da tecnologia ao longo do tempo. A camada de tecnologia foi subdivida em competências que existem e que precisam ser desenvolvidas, parcerias e também em pesquisa e desenvolvimento.

A última etapa contou com um workshop de quatro horas para integração das três camadas de mercado, produto e tecnologia. Neste workshop, também foram definidas ações para execução do roadmap. Essa etapa contou com a participação de 12 pessoas das áreas de engenharia, produto e assistência técnica e foram compilados os dados extraídos do workshop, tendo sido elaborado um relatório final e identificados os próximos passos para a área responsável executar as ações do roadmap. 


\section{$5 \quad$ Análise e discussão dos dados}

Para análise do conteúdo das entrevistas, foram utilizados diagramas para codificar seletivamente os conceitos e categorias identificadas (Strauss \& Corbin, 2008). As Figuras 2, 3 e 4 representam os diagramas conceituais resultantes da consolidação das entrevistas. Como o roadmapping adota uma abordagem bastante visual, os diagramas foram importantes tanto para a análise das entrevistas bem como para elaboração da síntese dos resultados.

A Figura 2 consolida o entendimento do conceito de roadmapping pelos entrevistados, os benefícios percebidos, a ligação do roadmapping com o planejamento estratégico e com a inovação bem como a construção da camada de mercado. O roadmapping construído na empresa gerou um mapa com a visão de futuro, em consonância assim com a revisão de literatura. Curiosamente, os funcionários entrevistados perceberam a contribuição da ferramenta roadmapping para estratégia, porém ainda é incipiente a percepção sobre o conceito do grupo para a dimensão de inovação do roadmapping. Segundo os entrevistados, o enfoque foi maior na perspectiva estratégica, sendo que a perspectiva da inovação não foi tão evidenciada, pois o tema escolhido foi muito amplo e englobava muitas questões. Por se tratar de um projeto piloto de roadmapping, os resultados fazem crer que se torna necessário fazer mais projetos para que as pessoas percebam mais facilmente os aspectos correlatos à inovação a partir do mapa. No âmbito da organização estudada, confirmou-se que o roadmapping construído teve um caráter amplo e mais similar ao strategic roadmapping do que propriamente ao technology roadmapping.

Entre os benefícios verificados, os resultados ressaltaram a integração entre as áreas da empresa que possuem certa dificuldade para sair da rotina e conversar entre elas. Outro benefício foi o alinhamento de conhecimento entre os participantes. Conforme Phaal et al. (2010), são necessários, para um bom desenvolvimento do processo de roadmapping, ter desejo de desenvolver processos eficazes, ter as pessoas certas envolvidas e o comprometimento da alta direção.

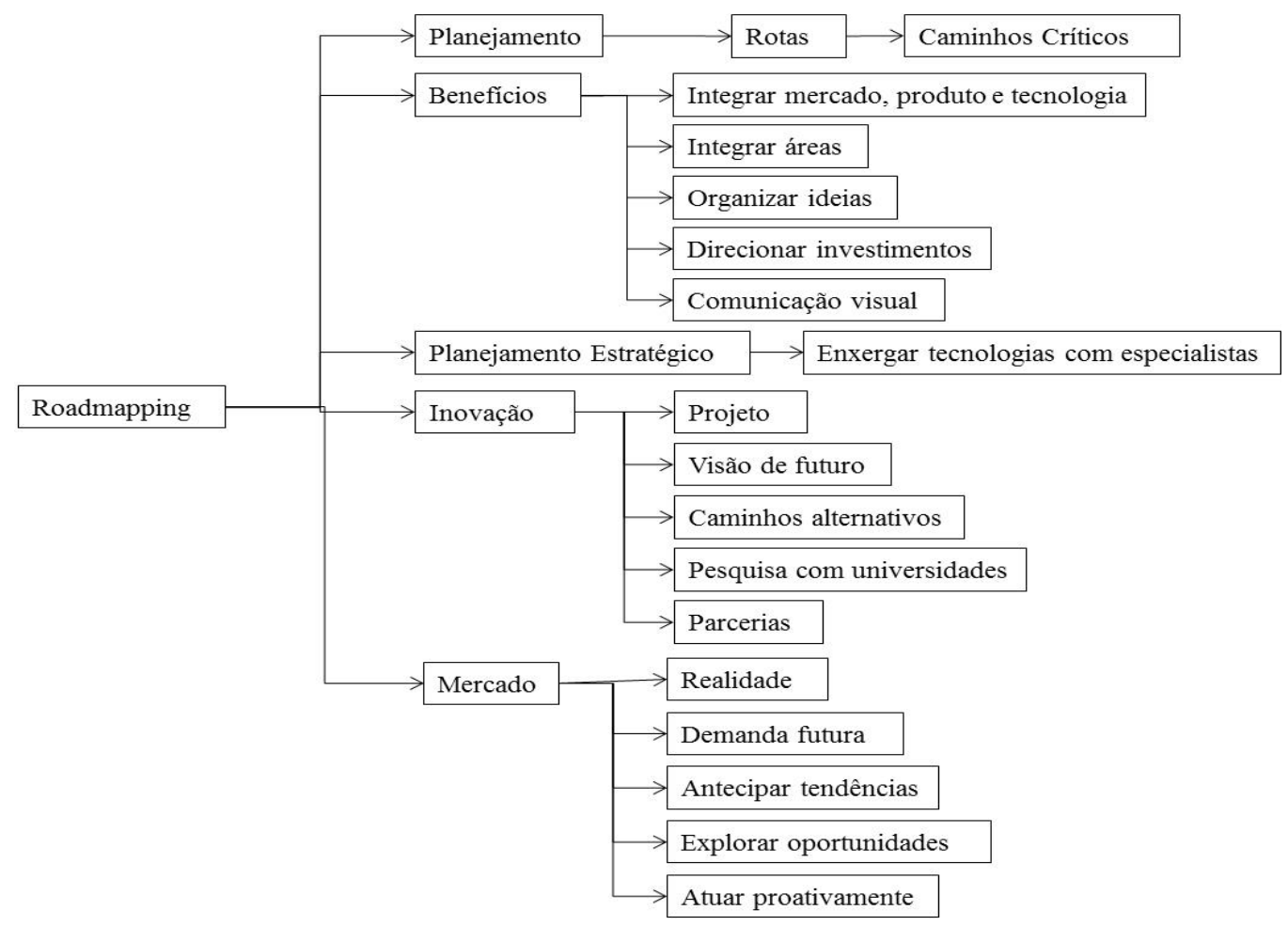

Figura 2: Contribuições do roadmapping em planejamento estratégico, inovação e mercado.

Fonte: Elaborado pelos autores a partir dos dados da pesquisa. 
A Figura 3 mostra tanto as contribuições percebidas pelos entrevistados do roadmapping em relação às camadas construídas de produto, tecnologia, a dinâmica de construção da ferramenta bem as dificuldades do seu uso. Segundo as entrevistas e a observação participante, o envolvimento das pessoas poderia ter sido mais bem explorado no roadmapping construído. No treinamento sobre roadmapping participaram vinte e seis pessoas. No entanto, apenas a metade desse grupo conseguiu continuar a participar dos workshops devido à sobrecarga de tarefas diárias. Para outras pessoas, os workshops coincidiam de acontecer no dia de viagens ou tarefas externas.

A falta de preparação de algumas pessoas sobre a ferramenta, por não terem participado do treinamento, também dificultou um pouco os workshops, pois se repetia o trabalho anterior e a lógica do workshop em cada evento realizado. O interesse de alguns especialistas para preparação prévia dos workshops foi fundamental para o desenvolvimento dos trabalhos. Por outro lado, os demais profissionais que não fizeram as atividades prévias impactaram o trabalho do roadmap, pois se demandou mais tempo entre os workshops para a busca de informações e, nos próprios workshops, para se completar as atividades. Convém lembrar Phaal et al. (2010) que ressaltam que a eficácia do workshop é maximizada com trabalho prévio.

Adicionalmente, o apoio da alta direção precisa ser melhorado dentro da organização para o roadmapping, pois com tal apoio se consegue mais facilmente o envolvimento das pessoas. Para os entrevistados, é necessário melhorar o tempo entre cada workshop e mobilizar mais as pessoas para a participação, requerendo novamente o envolvimento da alta direção. Os workshops foram considerados longos pelo alto nível de conhecimento e hábito com ritmo ágil de trabalho por parte dos funcionários.

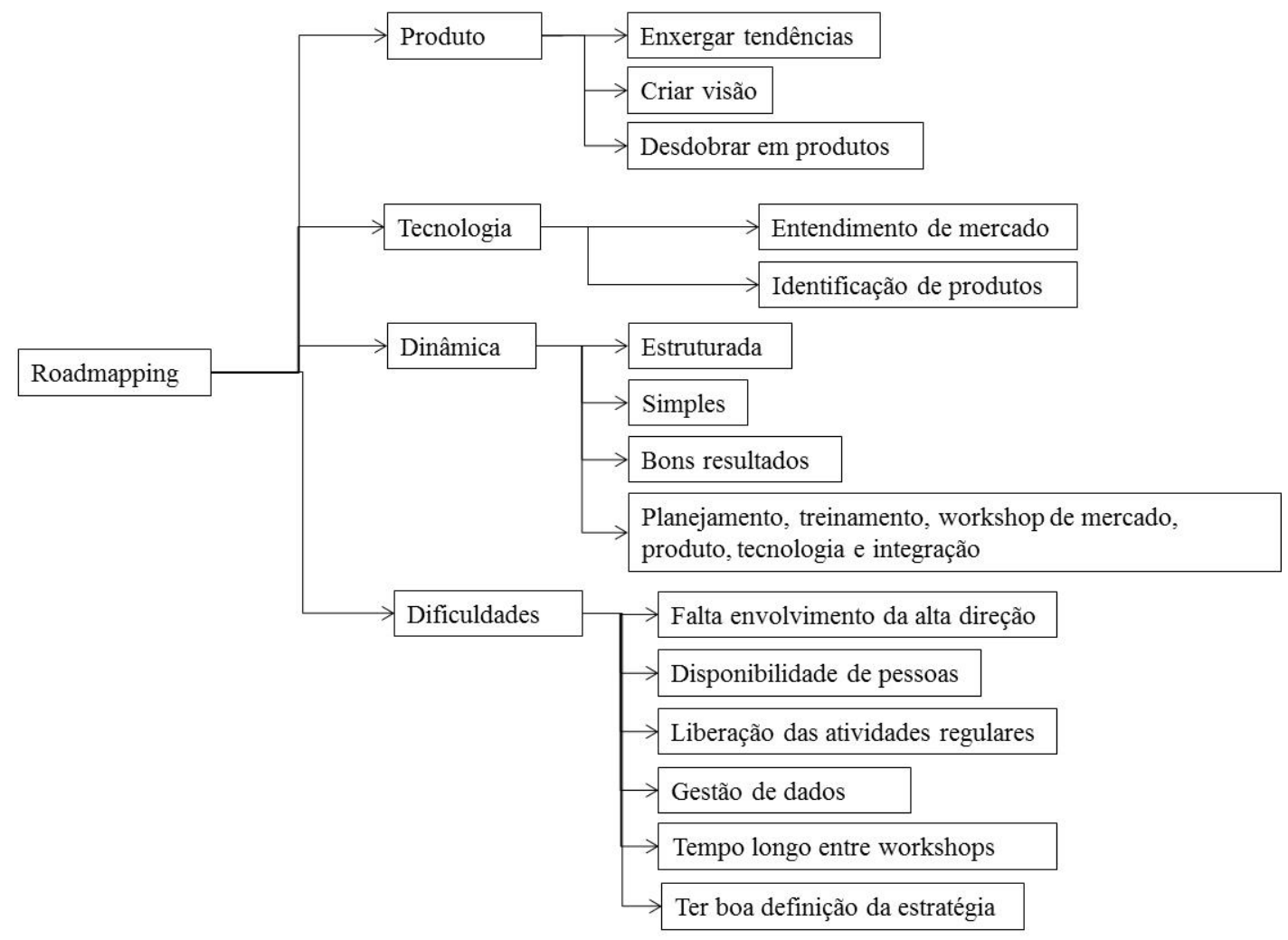

Figura 3: Contribuições do roadmapping em produto, tecnologia, dinâmica e dificuldades. Fonte: Elaborado pelos autores a partir dos dados da pesquisa. 
Os fatores que dificultam o roadmapping incluem a sobrecarga de iniciativas, distração de tarefas de curto prazo, falta de dados necessários e o conhecimento não estar disponível (Phaal et al., 2010). No caso estudado, a sobrecarga de iniciativas de curto prazo impactou os resultados do trabalho do roadmapping, pois muitos trabalhos são focados nos resultados e metas que o funcionário precisa atingir no ano. Além disso, a falta de dados foi verificada pela falta de disponibilidade de alguns especialistas em coletar dados previamente e participar dos workshops.

A Figura 4 sintetiza a percepção dos entrevistados sobre como o roadmapping auxilia a conexão e consolidação de informações e também sobre quais são os resultados efetivos do processo. A indústria automotiva estudada está começando a utilizar a ferramenta e já consegue visualizar os ganhos com o roadmapping, tais como mapeamento de tecnologias futuras sobre segurança veicular e a integração das pessoas de áreas diferentes. Antes da utilização da ferramenta, a empresa não conhecia nenhuma abordagem que permitisse, de uma forma visual, a integração das perspectivas da tecnologia com o mercado. Com o roadmapping, foi possível visualizar essa integração no assunto segurança veicular e ficou evidenciada a flexibilidade da ferramenta em lidar com outros conteúdos estratégicos.

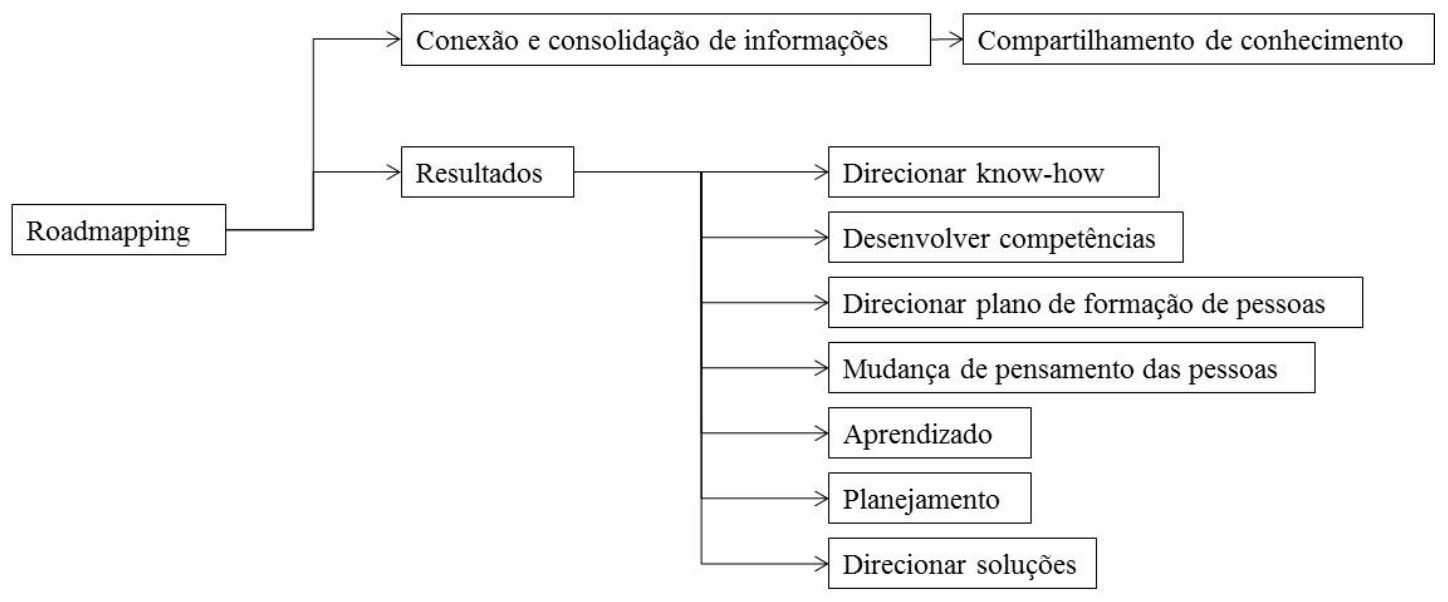

Figura 4: Contribuições do roadmapping para conexão e consolidação de informações e para geração de resultados

Fonte: Elaborado pelos autores a partir dos dados da pesquisa.

Portanto, com base nos dados coletados, recomenda-se adequar o processo para o uso prático da ferramenta no setor automotivo. Os workshops devem ter intervalos mais curtos entre as sessões e devem ter o apoio da diretoria para maior participação dos especialistas. Os principais resultados obtidos com a ferramenta compreendem o envolvimento de pessoas de diversas áreas e o próprio roadmap construído, que é um resultado bastante tangível que clarifica a área de segurança veicular, as lacunas tecnológicas e as tendências futuras da tecnologia. Além disso, conforme depoimentos dos entrevistados, o roadmapping aumentou o conhecimento dos envolvidos no assunto abordado, evidenciando a aprendizagem organizacional e o direcionamento de soluções tecnológicas.

\section{Conclusões}

Verificou-se que o conceito de roadmapping entre os participantes está bem claro como uma ferramenta para construção de rotas para prospectar o futuro, que dirige a estratégia da empresa e se desdobra em rotas tecnológicas. A partir da análise dos dados, foi possível observar que o roadmap 
contribuiu de fato para a inovação tecnológica ao mostrar o caminho ao longo do tempo que a empresa deve seguir para se atingir a estratégia. A integração de mercado, produto e tecnologia que a ferramenta proporciona foi um grande benefício percebido, além da integração das pessoas de diferentes áreas, conforme enfatizado pelos entrevistados. Outro grande benefício da ferramenta é direcionar os investimentos e permitir um melhor planejamento do resultado da empresa.

Constatou-se também que a contribuição da ferramenta é mais perceptível, em um primeiro momento, para o planejamento estratégico do que propriamente para a gestão da inovação. Essa percepção pode ser explicada em parte em razão de se ter tido um escopo amplo com o assunto segurança veicular, fazendo com que as discussões ficassem em um nível mais macro. Tal conexão entre o roadmap e a estratégia é prevista na literatura, corroborando assim os achados de Alcântara e Martens (2019), para quem a implementação do TRM como parte da estratégia de negócios é desafiadora, pois pode afetar o processo de trabalho, estrutura e cultura organizacional. Como lição aprendida para a organização, fica a possibilidade de se escolher escopos um pouco menores em futuras aplicações da ferramenta, permitindo assim uma maior profundidade tecnológica.

No caso estudado, o roadmap construído contribuiu para a gestão da inovação tecnológica, mas seu pleno potencial ainda não foi vislumbrado pela empresa. A técnica necessita ser mais utilizada e se deve começar a gerar os projetos com o roadmap para se enxergar a contribuição da ferramenta para a gestão da inovação. Por outro lado, as analistas de materiais e de negócios que fizeram novas utilizações dessa ferramenta, enxergam a ferramenta como uma forma de fazer a gestão da inovação tecnológica, pois conseguiram colocar os marcos ao longo do tempo e direcionar o trabalho e investimento para se obter uma vantagem estratégica para a empresa. O caso estudado confirma a afirmativa de Tidd, Bessant e Pavitt (2008) que consideram ser um grande desafio ter a definição estratégica correta para o desenvolvimento de trajetória tecnológica de uma empresa. Apesar dessa dificuldade, o roadmapping mostrou, para o caso estudado, ser uma ferramenta que auxilia a visualização dos principais vetores da trajetória tecnológica.

Outras recomendações futuras seriam estabelecer um elo entre o roadmap e os projetos da empresa, reduzir o escopo de futuras temáticas do roadmap, fazer revisões periódicas no roadmap construído de forma que ele fique sempre atualizado e também acoplar a ferramenta ao planejamento estratégico da empresa.

Verificou-se que a ferramenta integra as pessoas e as áreas envolvidas e também amplia a visão dos envolvidos de forma que direciona o know-how no assunto abordado, no caso em relação à segurança veicular. No caso estudado, o roadmapping direcionou os projetos da empresa e auxiliou na tomada de decisões. Além disso, o roadmapping permite uma visualização de ações que devem ser tomadas pela empresa ao longo do tempo, desdobrando bem o que a empresa deve fazer para se atingir os objetivos.

Enfim, quanto às contribuições deste trabalho, do ponto de vista profissional, ele poderá contribuir para a compreensão da utilização do roadmapping no setor automotivo, a partir da percepção dos profissionais que já participaram do processo dentro da empresa. As informações geradas pelo resultado poderão facilitar o conhecimento e a importância da ferramenta dentro da organização. Além disso, acredita-se que o trabalho possa facilitar a visão estratégica da empresa e a priorização de projetos, principalmente os de base tecnológica. Observou-se também que se deve trabalhar a forma de motivação dos funcionários para melhorar a condução da metodologia na empresa e melhorar o resultado do roadmap.

Esse trabalho mostrou o roadmapping como ferramenta que propicia a gestão do conhecimento, por meio da externalização do conhecimento dos especialistas para o roadmap e também por meio da socialização entre os especialistas, podendo ser fonte de estudos futuros para 
verificação da abordagem da Gestão do Conhecimento com o roadmapping.

Faz-se pertinente ressaltar as limitações do presente estudo. Esta pesquisa buscou a compreensão do roadmapping em apenas uma planta industrial situada no Estado de Minas Gerais de empresa multinacional do segmento automotivo. Foi dada ênfase no trabalho ao projeto piloto de segurança veicular, sendo que atualmente já existem novos casos de aplicação da ferramenta na empresa. Foram selecionadas para a entrevista desse trabalho as pessoas que mais participaram dos workshops para a construção do roadmapping bem como as pessoas que estão difundindo a ferramenta na empresa, resultando na limitação de treze pessoas entrevistadas. Não foram investigados a compreensão, a visão sobre o uso e os benefícios da ferramenta roadmapping na perspectiva da alta direção da empresa. Também não se averiguou a percepção da alta cúpula sobre como os resultados do roadmapping podem impactar na definição de objetivos e metas corporativas.

A fim de se aumentar o número de estudos sobre o tema, recomenda-se que esta pesquisa seja expandida para outras empresas fora do ramo automobilístico. Para empresas do setor automotivo, sugerem-se estudos de caso múltiplos de forma a contemplar a variação da ferramenta roadmapping.

Recomenda-se ampliar o estudo com um enfoque longitudinal, abordando os resultados do roadmap em um horizonte temporal mais amplo, uma vez que nem todos os frutos do roadmapping foram colhidos já que os resultados de inovação tecnológica demoram a ser efetivados. Outra sugestão consiste em abordar o papel da liderança, uma vez que na literatura, esse papel consta como primordial para o roadmapping.

Os trabalhos relacionados às aplicações do roadmapping são escassos na literatura nacional e por ser uma técnica eficaz para as organizações, os estudos de casos auxiliam a visualizar as adaptações dessa ferramenta em cada contexto. Nessa pesquisa, conseguiu-se compreender a utilização do roadmapping no setor automotivo, seus benefícios, limitações e oportunidades de melhoria. A partir desse trabalho, podem-se aprofundar estudos sobre a questão do papel da liderança no roadmapping, e na motivação dos envolvidos na aplicação da ferramenta. Além disso, acredita-se que a contribuição teórica do presente trabalho é suscitar a reflexão sobre interfaces entre os campos da estratégia e da inovação que são evidenciados pelo emprego da ferramenta de roadmapping.

\section{Referências}

Abe, H., Suzuki, A., Etoh, M., Sibagaki, S., \& Koike, S. (2008). Towards systematic innovation methods: innovation support technology that integrates business modeling, roadmapping and innovation architecture. In: PICMET, pp. 2141-2149.

Alcântara, D. P. \& Martens, M. L. (2019). Technology Roadmapping (TRM): a systematic review of the literature focusing on models. Technological Forecasting and Social Change, 138, 127-138.

Agência Brasileira de Desenvolvimento Industrial. Brasil Maior. Agendas Estratégicas Setoriais. (2013). 139 pp. Recuperado de http://www.brasilmaior.mdic.gov.br/images/data/201304/d874d3cdbd3a7e5d9cf32a28 a3b083b0.pdf. Acesso em 31 de dezembro de 2013

ANFAVEA. (2020). Anuário da Indústria Automobilística Brasileira. Recuperado de http://www.anfavea.com.br//anuario2020/anuario.pdfl.

Bray, O. H., \& Garcia, M. L. (1997). Technology roadmapping: the integration of strategic and technology planning for competitiveness. Innovation in Technology Management - The Key to Global Leadership. In: PICMET: Portland International Conference on Management and Technology, 25-28.

Daim, T., \& Oliver, T. (2008). Implementing technology roadmap process in the energy services sector: A case study of a government agency. Technological Forecasting and Social Change. 75(5), 687-720. 
Eisenhardt, K. M. (1989). Building Theories from Case Study Research. Academy of Management Review. $14(4), 532-550$.

Echols, A. E., \& Petrick, I. J. (2004) Technology roadmapping in review: a tool for making sustainable new product development decisions. Technological Forecasting and Social Change, 71(1), 81-100.

Hussain, M., Tapinos, E., \& Knight, L. (2017) Scenario-driven roadmapping for technology foresight. Technological Forecasting and Social Change, 124, 160-177

Kostoff, R. N., \& Schaller, R. R. (2001). Science and Technology Roadmaps. In: IEEE Transactions on Engineering Management, 48(2), 132-143.

Phaal, R.; Farrukh, C. J. P., \& Probert, D. R. (2004). Technology roadmapping - A planning framework for evolution and revolution. In: Technological Forecasting and Social Change, 71, 5-26.

Phaal, R., Farrukh, C. J. P., \& Probert, D. R. (2007). Strategic Roadmapping a workshop-based approach for identifying and exploring strategic issues and opportunities. In: Engineering Management Journal, 19(1), 3-12.

Phaal, R., Farrukh, C. J. P., \& Probert, D. R. (2010). Roadmapping for strategy and innovation. Aligning technology and markets in a dynamic world. University of Cambridge.

Phaal, R., Farrukh, C., \& Probert, D. (2001). Technology Roadmapping: linking technology resources to business objectives. University of Cambridge. 1-18.

Rothwell, R. (1994). Towards the Fifth-generation Innovation Process. International Marketing Review. MCB University Press. 11(1). pp. 7-31.

Strauss, A., \& Corbin, J. (2008). Pesquisa Qualitativa. Técnicas e procedimentos para o desenvolvimento de teoria fundamentada. São Paulo: Bookman.

Serva, M., \& Júnior, P. J. (1995). Observação participante e pesquisa em administração. Uma postura antropológica. Revista de Administração de Empresas, 35(1), 64-79. São Paulo.

Tidd, J.; Bessant, J.; Pavitt, K. (2008). Gestão da Inovação. Porto Alegre: Bookman.

Willyard, C. H., \& McClees, C. W. (1987). Motorola's Technology Roadmap Process. Research Management. $30,13-19$. 